\title{
Reducing Overheads for Fault-tolerant Datapaths with Dynamic Partial Reconfiguration
}

\author{
James J. Davis and Peter Y. K. Cheung \\ Department of Electrical and Electronic Engineering \\ Imperial College London \\ London, SW7 2AZ, United Kingdom \\ E-mail: $\{$ james.davis06, p.cheung $\} @$ imperial.ac.uk
}

\begin{abstract}
As process scaling and transistor count inflation continue, silicon chips are becoming increasingly susceptible to faults. Although FPGAs are particularly susceptible to the effects of such scaling, their runtime reconfigurability offers unique opportunities for fault-tolerance. This work presents an application combining algorithmic-level fault detection with dynamic partial reconfiguration to allow faults manifested within its datapath at runtime to be circumvented.
\end{abstract}

Keywords-Algorithm-based fault-tolerance, dynamic partial reconfiguration, error recovery, matrix multiplication.

\section{INTRODUCTION}

By tailoring fault-tolerant hardware to the application it protects, it is possible to reduce overheads without causing correspondingly large decreases in fault vulnerability. Algorithm-based fault tolerance (ABFT), applicable to a wide range of linear algebra operations [1] represent established methods for achieving such ends. Originally conceived for many-core applications, ABFT has been implemented in FPGAs to realise hardened matrix multiplication designs [2]. Our recent work [3] used the same operator as a case study for the implementation of a complete fault tolerance system, using ABFT for error detection and additional logic for subsequent fault avoidance.

Dynamic partial reconfiguration (DPR) has been explored as a means for facilitating runtime fault avoidance-exploiting reconfigurability to work around faulty components-in the past. Such non-application specific schemes, however, suffer from either high detection latency or low fault tolerability [4].

Here, we revisit ABFT-protected matrix multiplication, using DPR in place of additional logic to route around faults.

\section{ERror Detection And FAult Avoidance}

Central to this work is a hardware matrix multiplier, unrolled to perform computations on entire rows in parallel, implemented on a Xilinx Zynq system-on-chip. The checksum generation and verification required for ABFT [3] is performed by 'bolt-on' logic added to the input and output sides of the datapath, while the datapath itself is expanded by one multiply-accumulator (MAC) to mirror the matrices' expansion. What is most attractive about ABFT in this case is that the error detection circuitry represents a one-off fixed cost; the proportional area overhead incurred through its addition decreases as the problem size increases.
Faults that occur within the datapath can be attributed to particular MACs thanks to the fact that each is used to calculate the elements of exactly one output matrix column. Multiple faults result in checksum mismatch combinations that are able to identify faulty MACs simultaneously.

Since identical parallel MACs are used to perform complete multiplications, the mapping of those units to the output matrix columns they represent is inconsequential to the results. For this reason, once one or more are diagnosed as faulty, remaining healthy units can be substituted-multiple times per operation if necessary-for them. Such action has the effect of reducing parallelism to maintain accurate computation at the expense of increased runtime.

Fed with ABFT hardware-obtained fault location information, our system uses DPR to apply appropriate partial bitstreams, each representing a different routing configuration for the input and output side of the datapath, necessary to route data around faulty MACs. Since relatively few nets are affected, the partial bitstreams are small and consequently take only tens of to a few hundred $\mu$ s to apply.

\section{CONCLUSION}

Our experiments have shown the combination of ABFT and DPR to be effective in creating robust hardware with low overheads. When compared with our previous work, the replacement of dedicated rerouting logic with DPR resulted in an area overhead reduction from $17.3 \%$ to $9.01 \%$ for our largest tested design. In the future, our ABFT-based work will focus upon its application at differing levels of precision and expansion to additional operators.

\section{REFERENCES}

[1] K.-H. Huang and J. Abraham, "Algorithm-based fault tolerance for matrix operations," IEEE Transactions on Computers, vol. C-33, no. 6, pp. 518-528, 1984.

[2] A. Jacobs, G. Cieslewski, and A. George, "Overhead and reliability analysis of algorithm-based fault tolerance in FPGA systems," in International Conference on Field Programmable Logic and Applications (FPL), 2012, pp. 300-306.

[3] J. Davis and P. Cheung, "Datapath fault tolerance for parallel accelerators," in Field-Programmable Technology (FPT), 2013 International Conference on, Dec 2013, pp. 366-369.

[4] E. Stott, P. Sedcole, and P. Cheung, "Fault tolerance and reliability in field-programmable gate arrays," Computers Digital Techniques, IET, vol. 4, no. 3, pp. 196-210, May 2010. 